\title{
LAS IMPLICACIONES FAMILIARES EN LOS CUIDADOS DE LOS INMIGRANTES
}

\author{
Manuel Moreno Preciado \\ Profesor Titular Universidad Europea de Madrid \\ Doctor en Antropología de la Salud
}

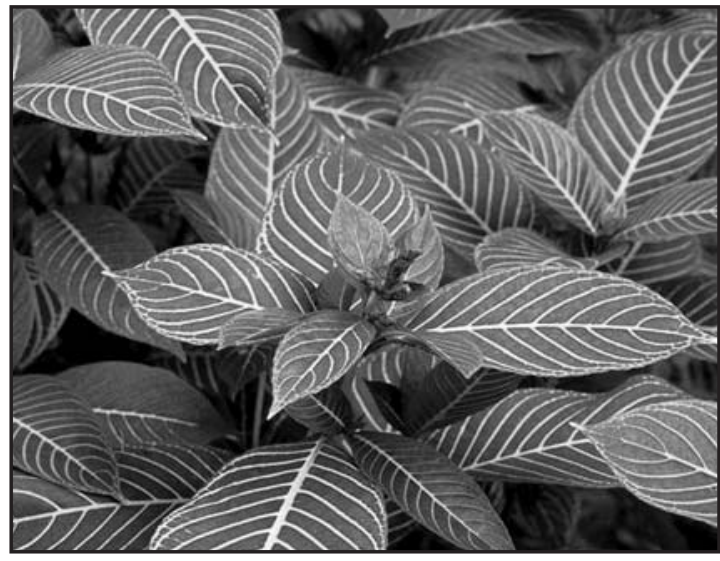

FAMILY INVOLVEMENT IN IMMIGRANTS HEALTH CARE

\section{SUMMARY}

$\mathrm{T}$ The present study is an approach to health care in immigrants and their domestic environment, obtained by means of experience gathered in the latest years. In the use of social and cultural factors applied to processes of health/sickness/attention, the anthropological view has been of more help in some cases than others circumstance that can be confirmed in those cases related to cultural diversity: food habits, costume, language barriers, religious practices, controversial traditions... etc...

At the same time some other interesting factors as social conditions within the immigrant context have been neglected.

This sort of biased view offers an out of context analysis concealing the imminent social etiology of health problems in those immigrant communities as recently paramount studies have shown. Thus, a change of direction is needed, a direction that should focus the studies in the cross-road of two processes or ways: the immigrant process and the health/sickness/attention process. The strong cultural tendencies predominant in medical professionals could only be overcome when there be a special education to promote cultural diversity according to what Edgar Morin proposes, an education focussed in human condition.

Key words: Immigration, family, health care, social and cultural factors.

\section{AS IMPLICAÇÕES FAMILIARES NOS CUI- DADOS DOS IMIGRANTES RESUMO}

\section{RESUMO}

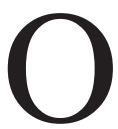
artigo é uma aproximação ao cuidado do imigrante e seu contexto familiar, através de uma análise da experiência acumulada nestes últimos anos.

No uso dos fatores sociais e culturais aplicados ao processo saúde-doença/ atenção, o olhar antropológico tem possibilitado mais uns processos que outros e isto se concretiza nos estudos sobre a população imigrada como uma tendência em direção a determinados processos relacionados com a diversidade cultural: hábitos alimentares e vestimentas, barreira lingüística, práticas religiosas, tradições controvertidas, etc. Ao mesmo tempo, constata-se um descuido de outros fatores como as condições sociais do contexto migratório. Este olhar preconceituoso está produzindo uma análise descontextualizada ao esconder a etiologia eminentemente social dos problemas de saúde da população imigrante, como evidenciado por recentes e importantes estudos.

Faz-se necessário uma "mudança de rumo" que centre estes estudos na análise da confluência dos processos ou itinerários: o processo migratório e o processo saúde-doença / atenção. As fortes tendências culturalistas que predominam nos profissio- 
nais de saúde só podem ser superadas a partir de uma educação para a convivência na diversidade cultural, que como disse Edgar Morin, deve ser uma educação centrada na condição humana.

Palavras-chave: Imigração; família; cuidado; fatores sociais e culturais.

\section{RESUMEN}

Este artículo es una aproximación al cuidado del inmigrado y a su contexto familiar, mediante un análisis de la experiencia acumulada en estos últimos años.

En el uso de los factores sociales y culturales aplicados al proceso salud/enfermedad/atención la mirada antropológica ha favorecido más unos procesos que otros y esto se concreta en los estudios sobre la población inmigrada en una tendencia hacía determinados procesos relacionados con la diversidad cultural: hábitos alimentarios e indumentarios, barrera lingüística, prácticas religiosas, tradiciones controvertidas, etc. Al mismo tiempo se constata un descuido de otros factores como las condiciones sociales del contexto migratorio. Esta mirada sesgada está produciendo un análisis descontextualizado al enmascarar la etiología eminentemente social de los problemas de salud de la población inmigrada, tal como lo evidencian recientes e importantes estudios.

Se hace necesario un "cambio de rumbo" que centre estos estudios en el análisis de la confluencia de dos procesos o itinerarios: el proceso migratorio y el proceso salud/enfermedad/atención. Las fuertes tendencias culturalistas que predominan en los profesionales sanitarios sólo pueden ser supera-

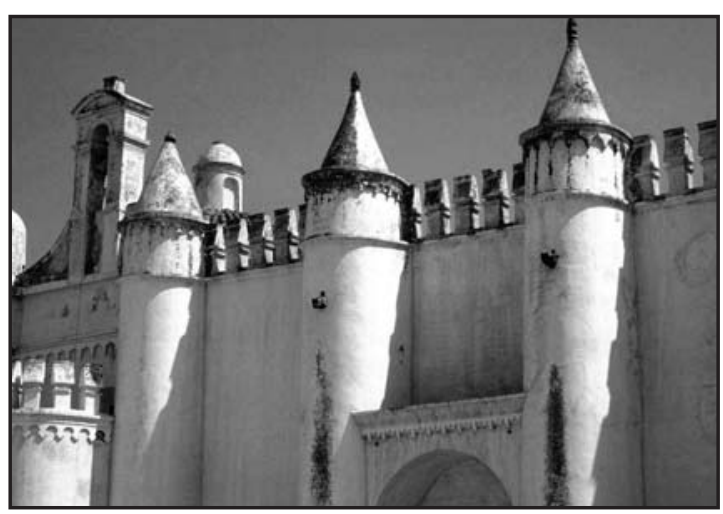

das desde una educación para la convivencia en la diversidad cultural, que como dice Edgar Morin, debe ser una educación centrada en la condición humana.

Palabras clave: Inmigración; familia; cuidado; factores sociales y culturales.

\section{Introducción. La mirada antropológica y el uso de los factores sociales y culturales}

La mirada antropológica consiste en las diferentes formas de observar y analizar los hechos sociales y culturales. Es sabido que la antropología tiene como objeto de estudio la cultura, su descripción e interpretación, Se trata, de una "descripción densa" (Gertz) que permita una reflexión sobre la naturaleza de los hechos sociales y culturales con objeto de comprender cómo la gente les hace frente. Evidentemente, los hechos sociales y culturales serán interpretados en función de los presupuestos teóricos con los que se realiza esta mirada: "Pensar, por ejemplo, -como continúa diciendo Wolcott- que la cultura se detecta mejor en lo que la gente hace, en lo que dice, en lo que dice que hace o en la tensión entre lo que hace y dice que debería hacer, supone inclinarse por una determinada concepción de la cultura...". (Jociles, 1999: 12).

Sobre el uso que se está haciendo desde la antropología de los factores sociales y culturales aplicados al proceso salud/enfermedad/atención, Eduardo Menéndez (2000) analiza cómo esa mirada exotizada sigue marcando la tendencia en los estudios sobre salud: "La antropología parece reconocer lo cultural más en unos factores y procesos que en otros, y así mientras que lo religioso o lo mágico aparecen aceptados unánimemente como fenómenos culturales, no pasa lo mismo con una parte de los factores referidos a los campos educativos, jurídico o de la salud...y es la no inclusión o secundarización de los factores de tipo político, y/o sobre todo de tipo económico, cuando se describen e interpretan procesos y factores culturales referidos al proceso salud/enfermedad/atención” (2000: 166). Sostiene Menéndez que cómo consecuencia de esa ascendencia culturalista se están realizando enfoques descontextualizados que 
introducen sesgos importantes en los estudios antropológicos sobre salud. Así, apunta que: "siguen describiéndose síndromes culturalmente delimitados, 'mal de ojo', 'susto' o 'empacho', sin dar datos, no ya en términos de tasas de mortalidad, sino en términos del número de casos de mortalidad o de enfermos, así como de toda otra serie de rasgos significativos en términos de epidemiología sociocultural". (2000: 166).

Comparto este análisis de Menéndez y pienso que explican bien los planteamientos que están apareciendo en el ámbito específico de algunos estudios relacionados con la salud de la población inmigrada: "El auge de los discursos culturalistas ha impregnado el debate en torno a la inmigración. Los conceptos de diversidad cultural y de multiculturalismo han acaparado el interés de las instituciones y de los profesionales sanitarios derivando sus preocupaciones hacia las diferencias culturales: hábitos alimentarios e indumentarios, barrera lingüística, prácticas religiosas, tradiciones controvertidas, como la ablación del clítoris, etc.". (Moreno, 2008: 53). Para evitar esta mirada sesgada Menéndez propone situar las definiciones en base a la experiencia empírica (etnográfica) y encuadrar estas experiencias dentro de los procesos históricos, políticos y sociales.

\section{Análisis antropológico de los fenómenos migratorios}

España forma parte de ese tipo de sociedades denominadas abiertas (Popper, 1994) receptoras de personas procedentes de diferentes horizontes. Ha pasado de ser un país de emigrantes a ser un país de inmigrantes. La explicación está en el profundo cambio político y social que ha vivido en las últimas décadas. No obstante, es preciso señalar que el rechazo al extranjero es universal. El otro siempre ha provocado recelos, cuando no miedo. El ser humano, se dice, es un animal de costumbre, al que le cuesta salir de las rutinas establecidas, y al que toda novedad le produce una sensación de inseguridad. Así, todo lo nuevo despierta sospecha, como que una amenaza se cierne sobre nosotros: "Sin embargo, a pesar del carácter común de esa experiencia -o puede que a causa de ello-, todos los colectivos tienden a sancionar y excluir al extraño...Su condición de otro, de ajeno a lo que se considera propio -que no deja de ser aquello de lo que uno se apropia- hace que a lo largo de la historia el extranjero, figura radical de la otredad, arrastre siempre una oscura sombra de amenaza que reverbera en hostilidad y rechazo". (Criado, 2001: XII-XIII).

En estos momentos estamos asistiendo en el mundo a una recrudescencia de la estigmatización del inmigrante. Tenemos el ejemplo de lo que está sucediendo en Italia y también los acontecimientos en Sudáfrica con los brutales asesinatos de personas procedentes de otros países más empobrecidos, como Zimbabue o Mozambique. Aunque en España no se hayan producido incidentes de esta envergadura -ni siquiera tras los atentados del 11M- el asunto es serio y nos concierne directamente, porque ahora nuestro país ha entrado en una fase económica menos favorable que puede constituir un caldo de cultivo ideal para que los vendedores de miedos conviertan al inmigrado en chivo expiatorio de las dificultades sociales de la población.

\section{Inmigración y salud: dos procesos entrela- zados}

Indagar acerca de los factores sociales y culturales de la atención sanitaria a los inmigrados requiere un análisis previo del contexto en el que se inscriben esos factores. Detecto, de entrada, dos grandes ejes: el contexto migratorio y el contexto sanitario. Ambos contextos tienen un carácter dinámico, por lo que es frecuente referirse a ellos como procesos. Así, Oriol Romaní los denomina "itinerarios migratorios e itinerarios de salud" (2002:501). Entiendo la migración como un proceso donde la condición de inmigrado se pierde cuando la sociedad autóctona deja de proyectar en el sujeto imágenes simbólicas negativas; esto es cuando se ha convertido en uno más dentro de la sociedad autóctona; se le puede ver como diferente, sí, pero no en el sentido etnocentrista que la antropología confiere al concepto de la otredad. Este proceso puede conllevar varios años, dependiendo de la dimensión del cambio experimentado por el migrante y del estado de los mecanismos de adaptación a dicho cambio. La mayor o menor presencia de factores como distancia cultural, formación, empleo, entorno familiar, redes de apoyo, 
salud, rechazo social, etc., prolongarán o acortarán el periodo de transición del "otro" versus "nosotros".

El proceso migratorio puede visualizarse como formando parte de los ritos de paso -rites de passage- de un periodo liminal, donde en su fase central se produce una situación de umbral, es decir, que el migrante se encuentra en tierra de nadie: entre lo uno y lo otro. Los problemas asociados a esta fase son:, tristeza, soledad -la vida de los inmigrantes es a menudo una historia de hombres y mujeres solos, exclusión, desvalorización, desarraigo, duelo, anomia, etc., una combinación de estrés y duelo que ha sido definida por Joseba Achotegui (2002) como "Síndrome de Ulises". Estos dos factores interactúan entre sí, produciendo una tensión característica en los procesos migratorios. Es la búsqueda de un balance positivo entre lo que se gana y lo que se pierde. En la medida en que el migrante encuentra que la experiencia ha valido la pena, el duelo se va superando y el estrés va cediendo. Se trataría entonces de una situación normal y propia de todo proceso migratorio. Como señala Achotegui, esta situación es la mayoritaria porque la migración, en general, significa para el migrante una solución a sus problemas. Por el contrario, se dan situaciones en las que el migrante se encuentra con tal cúmulo de dificultades que su proyecto se verá muy comprometido e incluso abocado al fracaso. Aparece entonces el "duelo complicado", también definido por Achotegui como "síndrome del inmigrante con estrés crónico, múltiple y extremo".

Siempre me ha llamado la atención la escasez de datos sobre el perfil epidemiológico de la población inmigrada. Es poco frecuente que las detalladas memorias anuales de las instituciones sanitarias (hospitales, atención primaria, consejerías de salud, etc.,) incluyan datos al respecto. Por este motivo me ha parecido muy oportuno que la Encuesta Nacional de Salud, realizada en el año 2006 por el Instituto Nacional de Estadística y cuyos datos han sido publicados en marzo de 2008 haya tenido en cuenta, por primera vez, el factor "extranjero". Recojo en el cuadro $\mathrm{n}^{\circ} 1$ algunos de esos datos, que, en gran medida, refuerzan las tesis de quienes entendíamos que los problemas de salud de esta población se relacionan estrechamente con los de su condición de migrantes. Dice la encuesta que su salud no difiere sensiblemente de la salud de la población autóctona y las diferencias están relacionadas con el factor edad, género y sobre todo condiciones sociales y laborales. Así, su percepción del estado de salud es ligeramente mejor que el de la población autóctona; tienen menos enfermedades crónicas y sus hábitos de salud son similares. A pesar de que machaconamente se transmite la idea de que el migrante es alto consumidor de servicios sanitarios, no hay datos concluyentes al respecto y los pocos estudios serios realizados apuntan en la dirección contraria. Así, el informe del INE concluye que los inmigrados utilizan menos, por lo general, los servicios sanitarios que la población autóctona. Sólo en el caso de la visita a la matrona el porcentaje es superior al de los autóctonos, lo cual se explica por el alto componente de mujeres en edad fértil; el resto de los profesionales sanitarios reciben menos visitas. Puede decirse, entonces, que la salud de los inmigrados está condicionada, fundamentalmente, por las formas de vida asociadas al proceso migratorio.

\section{Inmigración y cuidados: repercusiones fami- liares}

El difícil acceso, por parte de los inmigrados, a los dispositivos sanitarios, es una referencia constante tanto desde las instituciones sanitarias y sus profesionales, como desde los propios inmigrados y sus asociaciones (Fernández-Rufete, 2004; Sanz et alt., 2004).

Según los profesionales sanitarios y los expertos, lo que dificulta la adaptación de los inmigrados a las normas de los dispositivos sanitarios es su difícil contexto sociolaboral. También se alegan factores culturales: "su reloj es distinto". Se apunta a la existencia de dos tipos extremos de usuarios del sistema sanitario: los que "no lo usan" y los que "lo usan frecuentemente". Entre los que "no lo usan", se encuentra un perfil de inmigrado, varón y con situación de irregularidad y/o precariedad sociolaboral. Entre los que usan con profusión los servicios sanitarios está el perfil de mujer, joven y con cierto nivel de asentamiento. Las enfermeras y matronas refieren cómo, una vez que estas personas entran en el circuito de acceso a los centros de salud, mediante la obtención de la tarjeta sanitaria, 
es frecuente que se familiaricen con estos servicios. Cabe en este punto plantear un interrogante: ¿quién es mejor/peor usuario de los dispositivos sanitarios, el que "usa" o el que "no usa"?

Hablar de cuidados y familia lleva directamente a hablar de niños. Como he apuntado antes en relación a las necesidades de salud de la población inmigrada, el contexto materno-infantil es donde más demandas de atención sanitaria se producen. Esto se debe al carácter femenino de la inmigración y a su juventud, lo que contrasta con la situación de la población autóctona. $\mathrm{M}^{\mathrm{a}}$ Ángeles Durán, investigadora del CSIC, en su libro El valor del tiempo sitúa la importancia de este factor demográfico: "El dato más importante sobre la infancia en la España actual es la disminución del número de niños y su llegada por planificación voluntaria; y el segundo, el crecimiento del número de niños venidos de fuera en compañía de sus padres, o nacidos en España de madre extranjera. Quince de cada cien niños nacidos vivos en 2005 son hijos de madre extranjera, pero la cifra es más alta si se añaden las madres que han obtenido la nacionalidad recientemente por matrimonio o residencia". (2007: 51). Pienso que estos datos nos ayudan a comprender las particulares demandas de cuidados de la población inmigrada.

La aparición de la enfermedad y/o la necesidad de frecuentar los dispositivos sanitarios provocan, en el inmigrado, un aumento del nivel de estrés. La causa principal es el riesgo de fracaso del proyecto migratorio: "aquí hemos venido a trabajar". Cuando acaece la enfermedad o el accidente, dependiendo de la gravedad del caso, se producen situaciones que son vividas con desasosiego por los pacientes y por sus familiares: "Los inmigrados han expresado con frecuencia sentimientos de tristeza que se relacionan con la dureza del contexto social en el que viven y con el alejamiento de los suyos... Una cierta dignidad les impide la manifestación de estos sentimientos, pero cuando la enfermera lo facilita, rápidamente salen hacía fuera. Son significativas dos expresiones empleadas por dos enfermeras: 'son muy estoicos' y 'cuando me siento a su lado enseguida tiro de clinex'...". (Moreno, 2008: 198).

El desconocimiento del funcionamiento de los dispositivos sanitarios -por parte de los inmigra- dos- y de los usos de salud -por parte de los profesionales- crea incomprensiones que afectan a la relación profesional/paciente. En este sentido expongo las manifestaciones, a modo de ejemplo, de dos mujeres marroquíes (Yasmina, mediadora intercultural y Saïda, joven paciente) expresadas en una entrevista conjunta (Moreno, 2008: 161162):

Yasmina: “...a veces me llaman las enfermeras, o un ginecólogo para ver por qué la mujer marroquí no cuida a su hijo como ellos quieren...jcómo que, qué en su país que no cuidan a su hijo!...es que alli hay unos cuidados específicos, distintos...sobre todo para la mujer que da a luz...eh..., allí toda la sociedad está volcada con ella 'no te muevas, estate quieta...que no hagas nada... que tiene que estar en la cama descansando...te llevan hasta la comida...hasta los calditos calentitos...al niño también le cuidan...”.

Saïda: "Es que aquí la mujer tiene que levantar...no descansa nada...y te mandan a casa enseguida...tienes que salir e ir a su casa y a lo mejor las cosas a lo mejor no son adecuadas para una mujer...”.

Yasmina: "En Marruecos antes el parto se hacía en el domicilio, ahora ya se hace en los hospitales... [Entonces, ¿se ha roto eso que tú decías? ] No, se mantiene, por ejemplo cuando sale la mujer del hospital, ese ritual se sigue en la casa...los cuidados....el parto es como cosa de mujeres...las mujeres se ayudan en estas cosas...el hombre hace otras cosas...él se ocupa de muchas cosas...pero de fuera, compra las cosas...va al mercado...compra el cordero...esos días se necesitan comprar cosas...".

Saïda: "Si, es así...aunque ahora algunas cosas están cambiando...creo que si... lo que pasa que, bueno es lo que decíamos, que ahora la mujer trabaja más, entonces sale más, pero también se cansa más...la mujer trabaja más... ahora tenemos la carga de la casa y además el trabajo...bueno aquí no es como la mujer latina que trabajan muchas, la mujer magrebi trabaja menos...”.

En síntesis, ellas consideran que la mujer es apoyada por su entorno, que se vuelca en ella y la colma de atenciones. Aquí, sin embargo, compatibilizando vida laboral y tareas domésticas conside- 
ran que para algunas cosas han perdido. Informantes de otras nacionalidades han hablado, también, del mantenimiento de tradiciones en relación a la maternidad y el parto diferentes a las de la sociedad autóctona.

Cada ves son más frecuentes las alusiones en medios de comunicación sobre cómo la inmigración está sirviendo para paliar algunos de los graves problemas de la sociedad autóctona, como el de la baja natalidad. En este sentido es preciso señalar la importancia de la emergente figura del "inmigrante cuidador-contratado". Cada día son más los niños y ancianos cuidados por inmigrados, en el domicilio o incluso en el hospital. Pienso que este aspecto puede favorecer una imagen social más favorable de la inmigración, que sea vista, no como problema, sino como solución a problemas.

\section{Cambio de rumbo}

Quizá, el más importante reto que tiene la sociedad sea el de la convivencia en la diversidad. Aprender a vivir en la diferencia es una asignatura pendiente en un mundo empeñado en repetir los peores episodios de la historia de la humanidad, que siempre tuvieron como acompañante, cuando no como detonante, el deseo de aprovecharse de las diferencias para convertirlas en desigualdades. Hoy, en nuestras sociedades abiertas, el tradicional racismo de carácter biológico ha cedido el paso a otro más centrado en las diferencias culturales. Son denominados racismos de la vida cotidiana (Del Olmo, 2002). Educar para la convivencia en la diversidad cultural es una prioridad que debe, a mi entender, situarse en el centro del compromiso profesional. La ética del cuidado puede servir como instrumento teórico para preservar los valores del cuidado, entendido como aquél que sitúa al paciente como la razón de ser de la práctica profesional, como la referencia, el punto de partida y de llegada de los cuidados. Siguiendo este planteamiento de Collière (1993), al paciente no se le considera como un objeto portador de tal o cual enfermedad, sino que constituye la propia finalidad de los cuidados, que no toman sentido si no es a partir de él y de lo que representa en su entorno social. Señalo, en este sentido, la importancia de la mirada cercana al inmigrado para superar los estereotipos y evitar los prejuicios; en el cuidado de los pacientes la cercanía es un valor esencial. No es posible cuidar desde la frialdad de la distancia, porque cuidar es interrelacionarse con la persona cuidada. Evidentemente, se pueden aplicar tratamientos técnicos de igual forma que se aplican a los objetos inertes, pero el cuidado es otra cosa: "La superación de la lejanía espacial y de la distancia ética es fundamental en la acción de cuidar. No se puede cuidar de alguien desde la lejanía. Para cuidar de una persona enferma, de una persona anciana es preciso acercarse a ella, superar la lejanía, pero también y la distancia no es un concepto de tipo físico, sino de carácter ético". (Torralba, 1998: 342).

También se hace necesaria la ética de la esperanza de la que nos hablaba Victoria Camps, porque estamos asistiendo a un recrudecimiento de los discursos del miedo. Hay riesgos en la inmigración y dificultades, pero también hay beneficios. Pasar del miedo a la diversidad al valor de la diversidad es el compromiso necesario. El hecho de que España haya sido capaz de integrar a más de cuatro millones de personas en poco tiempo y sin grandes tensiones es un logro del que toda la sociedad española podría sentirse orgullosa y demuestra el importante lugar que España ocupa en la esfera mundial. Demos, pues, una oportunidad a la esperanza.

Termino con esta frase de Edgard Morin que sitúa bien lo que, a mi entender, debe ser la educación del futuro: "La educación del futuro debe ser una enseñanza fundamental y universal centrada en la condición humana. Estamos en la era planetaria y los seres humanos, dondequiera que estén, están embarcados en una aventura común. Es preciso que se reconozcan en su humanidad común y, al mismo tiempo, reconozcan la diversidad cultural inherente a todo lo humano". (Morín, 201: 57).

\section{Bibliografía}

- Achotegui, J. (2002). La depresión en los inmigrantes: una perspectiva transcultural, Barcelona, Ediciones Mayo.

- Collière, M.F. (1993a) [1982]. Promover la vida, Madrid, McGraw-Hill Interamericana.

- Criado, M ${ }^{\mathrm{a}}$.J. (2001). La línea quebrada. Historias de vida de migrantes, Madrid, Cees.

- Del Olmo, M. (2002). "El negocio de las diferencias. Una aportación teórica y metodológica al estudio del racismo, el prejuicio y la discriminación", en J. García y C. Muriel (eds.), La inmigración en España, 141-148, Volumen II, Granada, Laboratorio de Estudios Interculturales.

- Durán, Ma . Á. (2007). El valor del tiempo, Madrid, Espasa Calpe. 
- Encuesta Nacional de Salud. Año 2006. INE. [Documento en línea] http://www.msc.es/estadEstudios/estadisticas/encuesta Nacional/encuesta2006.htm [consulta: 1-08-2008].

- Fernández-Rufete, J. y Rico, J.I. (2005). "Los dominios simbólicos de la inmigración. La dialéctica entre las demandas de atención sociosanitaria y la estructura de los dispositivos asistenciales en el caso de los inmigrantes ecuatorianos en la Región de Murcia”, en J. Fernández y M. García (eds.), Movimientos migratorios contemporáneos, 267-284, Murcia, UCAM.

- Jociles, M M $^{\mathrm{a}}$.I. (1999). Las técnicas de investigación en antropología. Mirada antropológica y proceso etnográfico. Gazeta de Antropología [Publicación en línea]. Gazeta de Antropología, 15. http://www.ugr.es/ pwlac/Welcome.html [consulta: 22-05-2008].

- Menéndez, E. (2000). "Factores culturales. De las definicio- nes a los usos específicos", en E. Perdiguero y JM. Comelles (eds.), Medicina y cultura. Estudios entre la antropología y la medicina, 163-188. Barcelona, Bellaterra.

- Moreno Preciado, M. (2006). El cuidado del "otro". Un estudio sobre la relación enfermera/paciente inmigrado, Barcelona, Bellaterra.

- Morin, E. (2001). Los siete saberes necesarios para la educación del futuro. Barcelona, Paidós.

- Popper, K. (1994) [1957]. La sociedad abierta y sus enemigos, Barcelona, Paidós.

- Romaní, O. (2002). "La salud de los inmigrantes y la de la sociedad. Una visión desde la antropología”, FMC, Volumen 09, 07: 498-504.

- Torralba i Roselló, F. (1998). Antropología del cuidar, Barcelona, Fundación Mapfre.

Cuadro 1: percepción de salud de la población inmigrada

\begin{tabular}{|c|c|c|}
\hline INDICADOR & EXTRANJEROS & NACIONALES \\
\hline \multicolumn{3}{|c|}{ ESTADO DE SALUD } \\
\hline $\begin{array}{l}\text { Valoración del estado de salud percibida } \\
\text { (Bueno o muy bueno) }\end{array}$ & $73,17 \%$ & $69,57 \%$ \\
\hline Calidad de vida relacionada con la población infantil & $58,37 \%$ & $62,13 \%$ \\
\hline Infartos & $9,18 \%$ & $20 \%$ \\
\hline Dolor lumbar & $15,75 \%$ & $15,75 \%$ \\
\hline Diabetes & $1,56 \%$ & $4,27 \%$ \\
\hline Colesterol alto & $1,86 \%$ & $4,74 \%$ \\
\hline Úlceras de estómago o duodeno & $1,90 \%$ & $6,56 \%$ \\
\hline Cataratas $5,63 \%$ frente al $15,30 \%$. & $5,63 \%$ & $15,30 \%$ \\
\hline \multicolumn{3}{|c|}{ ESTILOS DE VIDA } \\
\hline Fumador diario & $25,68 \%$ & $26,57 \%$ \\
\hline Consumen alcohol en las 2 últimas semanas & $48.94 \%$ & $56,60 \%$ \\
\hline Horas de sueño & $7,73 \%$ & $7,77 \%$ \\
\hline $\begin{array}{l}\text { Ejercicio físico en el trabajo: } \\
\text { - Sentado la mayor parte de la jornada } \\
\text { - Tareas con gran esfuerzo físico }\end{array}$ & $\begin{array}{l}18,12 \% \\
8,64 \%\end{array}$ & $\begin{array}{l}34,93 \% \\
4,94 \%\end{array}$ \\
\hline Hace ejercicio en el tiempo libre & $57,40 \%$ & $60,96 \%$ \\
\hline No sucle desayunar nada & $4,36 \%$ & $2,72 \%$ \\
\hline Lactancia natural & $79,73 \%$ & $67,43 \%$ \\
\hline Tienen peso normal en relación a estatura & $58,07 \%$ & $53,45 \%$ \\
\hline \multicolumn{3}{|c|}{ UTILIZACIÓN SERVICIOS SANITARIOS } \\
\hline $\begin{array}{l}\text { Han utilizado algún servicio sanitario en las últimas } 4 \\
\text { semanas }\end{array}$ & $29,3 \%$ & $39,5 \%$ \\
\hline Visita al médico en las últimas 4 semanas & $1,08 \%$ & $1,15 \%$ \\
\hline Visita a la enfermera en las últimas 4 semanas & $2,5 \%$ & $4,8 \%$ \\
\hline Visita al fisioterapeuta en las últimas 4 semanas & $1,6 \%$ & $2,9 \%$ \\
\hline Visita a la matrona en las últimas 4 semanas & $3,9 \%$ & $1,4 \%$ \\
\hline Vista al especialista en las últimas 4 semanas & $0,46 \%$ & $0,55 \%$ \\
\hline $\begin{array}{l}\text { Realización de pruebas no urgentes en las últimas } 4 \\
\text { semanas }\end{array}$ & $12,3 \%$ & $15,8 \%$ \\
\hline Hospitalizaciones en los últimos 12 meses & $8,8 \%$ & $9.0 \%$ \\
\hline
\end{tabular}

Fuente: Instituto Nacional de Estadística. Encuesta Nacional de Salud, 2006 